\title{
The Effect of Node Density and Propagation Model on Throughput Scaling of Wireless Networks
}

\author{
Enrique Duarte-Melo, Awlok Josan, Mingan Liu, David L. Neuhoff, and S. Sandeep Pradhan \\ Electrical Engineering and Computer Science Department. \\ University of Michigan, Ann Arbor, MI 48109
}

\begin{abstract}
This paper derives a lower bound of the form $n^{\gamma-1}$ to the per-node throughput achievable by a wireless network when $n$ source-destination pairs are randomly distributed throughout a disk of radius $n^{\gamma}, 0<\gamma<1 / 2$ and propagation is modeled by an attenuation of the form $1 /(1+d)^{\alpha}, \alpha>2$.
\end{abstract}

\section{INTRODUCTION}

The pioneering work of Gupta and Kumar [1] has led to many studies of scaling laws for the asymptotically achievable throughput in wireless networks under a variety of network models and assumptions. Such scaling laws help us understand the fundamental performance limits of these networks and how efficiency changes as network conditions change. Some examples include [2] where the nodes are allowed to move; [3], [4], [5], where many-to-one type of communications is considered; [6], [5], where cooperative communication schemes are employed to improve network throughput; and [7], where scaling laws are derived using directional antenna assumptions.

All of these scaling results are highly dependent on the various assumptions made, such as on the network topology (e.g., planar, linear, ring, sphere, etc.), the purpose of the network (e.g., many-to-many vs. many-to-one communications), the physical layer models (e.g., different signal propagation and interference models), and the asymptotic density of nodes (e.g. increasing to infinity or remaining constant).

This paper focuses on two such aspects - the underlying model for signal propagation, and the asymptotic density of nodes. We focus on the many-to-many communications task. Specifically, a set of $n$ nodes are randomly distributed over some region $A$, and each node randomly chooses another node to whom to transmit data. For this task, [1] found the maximum throughput per node to be ${ }^{1} \Theta(1 / \sqrt{n \log n})$, assuming a $1 / d^{\alpha}$ propagation law with $\alpha>2$. This result is essentially independent of the density of the nodes. For example, it applies when the region $A$ is fixed and the density of nodes increases linearly with $n$, and when the density of nodes is fixed and area of $A$ increases linearly with $n$. On the other hand, Arpacioglu and Haas [8] showed that when the propagation model has the form $1 /(1+d)^{\alpha}, \alpha>2$, and the region $A$ remains fixed (so node density increases linearly with $n$ ), the maximum attainable throughput decreases dramatically

\footnotetext{
This work was supported by NSF grants CCF-0329715 and ANI-0238035.

${ }^{1}$ We use the notation $\mathcal{O}\left(f_{n}\right), \Omega\left(f_{n}\right)$ and $\Theta\left(f_{n}\right)$, in the conventional way, i.e., to characterize a quantity $x_{n}$ depending on $n$ for which there are finite constants $c_{1}>0$ and $c_{2}>0$ such that, respectively, $x_{n}<c_{2} f_{n}, x_{n}>c_{1} f_{n}$, and $c_{1} f_{n}<x_{n}<c_{2} f_{n}$, for all $n$.
}

to $\Theta(1 / n)$. At the same time, it is relatively easy to see that if the $A$ increases linearly with $n$ (so that node density remains constant), then even with their propagation model, the maximum throughput is, $\Theta(1 / \sqrt{n \log n})$, i.e. the same as for the $1 / d^{\alpha}$ propagation model. These results are summarized in the following table. One concludes that throughput is greatly dependent on the assumptions about propagation model and node density.

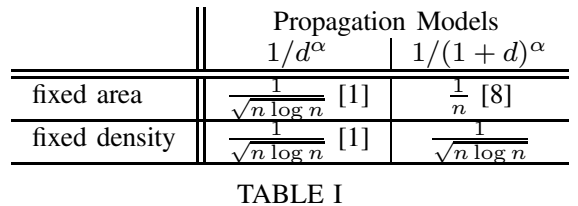

THROUGHPUT SCALING RESULTS UNDER DIFFERENT PROPAGATION MODELS AND NETWORK DENSITY ASSUMPTIONS

In this paper, we consider the maximum achievable throughput for the $1 /(1+d)^{\alpha}$ propagation model when the region $A$ grows at intermediate rates. Specifically, $A$ is chosen to be a disk of radius $n^{\gamma}, 0<\gamma<1 / 2$, and it is shown that throughput $\Omega\left(1 / n^{1-\gamma}\right)$ is attainable. Though we have not shown this to be the largest possible throughput, we suspect this to be the case. When $\gamma \approx 0$, the throughput $\Omega\left(1 / n^{1-\gamma}\right)$ approaches the $\Theta(1 / n)$ result found in [8]. For $\gamma \approx 1 / 2$, the throughput $\Omega\left(1 / n^{1-\gamma}\right)$ appears to be larger than the $\Theta(1 / \sqrt{n \log n})$ shown in the table for $\gamma=1 / 2$. To see what is happening, consider $\gamma=1 / 2-\epsilon$, where $\epsilon>0$ is very small. Our result says that when $n$ is large, the attainable throughput is at least $n^{-1 / 2-\epsilon}$, which is less than (not larger than) the $n^{-1 / 2}(\log n)^{-1 / 2}$ shown to be attainable in [1]. The appearance of a paradox is due to an implicit, but improper, interchange of the order of taking limits over $n$ and $\gamma$.

In the remainder of the paper, Section II introduces the many-to-many communication task and outlines the approach to the main result. Section III introduces propagation models and criteria for judging the success of a transmission. Section IV formalizes and outlines the proof of the main result. Due to space limitations, key lemmas are stated without proof. Section V summarizes.

\section{The Many-to-Many Communication TASK}

A set of $n$ nodes, $\Sigma_{n}=\left\{s_{1}, \ldots, s_{n}\right\}$, is distributed over a disk $A_{n} \subset \mathcal{R}^{2}$ with radius $n^{\gamma}$, called the network region, where $s_{i} \in A_{n}$ denotes the $i$ th node as well as its location, and where $\gamma, 0<\gamma<1 / 2$, is a fixed parameter that 
characterizes how the area of the disk and the density of nodes scale with $n$. Each node serves as a source of bits that it wishes to communicate to some destination. We use the terms source and node interchangeably. For each source $s_{i}$, another of the $n$ nodes, denoted $d_{i}$, is designated as the destination for its bits. As a result, there is a source-destination set $\mathcal{P}_{n}=\left\{\left(s_{1}, d_{1}\right), \ldots,\left(s_{n}, d_{n}\right)\right\}$ consisting of $n$ sourcedestination pairs, each representing a desired conversation. Note that a node may serve as the destination for more than one source.

Each of the $n$ sources has an infinite number of bits it wishes to communicate to its destination node, as quickly as possible. Communication uses simple multihop relaying with a time slotted system. We make the usual assumption that the sourcedestination set is random.

\section{A. System Definition}

We now describe the kind of system to be used for the many-to-many task. This is basically an explicit formalization of the kind of system that appears implicitly in prior work of others. There is a transmitter and receiver at each of the $n$ nodes. The antennas at each node are omnidirectional. All transmitters use the same power $P$, which we get to choose and which may depend on $n$ and the specific source-destination set $\mathcal{P}_{n}$. (However, we will see that when our system is optimized, the power can remain constant.) As mentioned earlier, transmissions occur in slots. We assume there is a fixed $W>0$ such that each transmitter can transmit at most one packet, consisting of $W$ bits, in one slot, regardless of $P, n$ or any other factors. Such transmissions are received throughout the network region $A_{n}$ in the presence of background noise with power $N_{o}$ and interference from other transmitters transmitting at the same time. As a result, the packets might or might not be successfully received by an intended receiver. Criteria for determining success will be introduced later.

To communicate bits from the sources to their destinations, each source-destination pair needs a route and a schedule. A route for source-destination pair $\left(s_{i}, d_{i}\right)$ is a finite sequence of hops, $h_{i}=\left(h_{i, 1}, \ldots, h_{i, J_{i}}\right)$, from $s_{i}$ to $d_{i}$ with the $j$ th hop of the route being a pair $h_{i, j}=\left(t_{i, j}, r_{i, j}\right)$ indicating that node $t_{i, j} \in \Sigma_{n}$ is to transmit bits originating at $s_{i}$ with the intention that they be receivedby node $r_{i, j} \in \Sigma_{n}$. The first hop has the form $h_{i, 1}=\left(s_{i}, t_{i, 1}\right)$, subsequent hops have $r_{i, j}=t_{i, j+1}$, and the last hop has the form $h_{i, J+i}=\left(t_{i, J_{i}}, d_{i}\right)$. Paths for different source-destination pairs may have different numbers of hops. The length of a hop $h=(t, r)$ is the Euclidean distance $\|t-r\|$.

A schedule for route $h_{i}=\left(h_{i, 1}, \ldots, h_{i, J_{i}}\right)$ is a sequence of positive integers $\sigma_{i}=\left(\sigma_{i, 1}, \ldots, \sigma_{i, J_{i}}\right)$ assigning a time slot to each hop of the route. Specifically, node $t_{i, j}$ makes its transmission of hop $h_{i, j}$ in time slot $\sigma_{i, j}$. Combining the notions of route and schedule, each source-destination pair $\left(s_{i}, d_{i}\right)$ is assigned a scheduled route $H_{i}=\left(\left(h_{i, 1}, \sigma_{i, 1}\right), \ldots,\left(h_{i, J_{i}}, \sigma_{i, J_{i}}\right)\right)$.

We now define a system $\mathcal{S}_{n}$ for source-destination set $\mathcal{P}_{n}$ to be a set of $n$ scheduled routes $\left\{H_{1}, \ldots, H_{n}\right\}$. Such a system is assumed to operate periodically with period $p=$ $\max _{i, j} \sigma_{i, j}$, which is the largest slot assignment of any hop of any route. That is, the $j$ th hop of route $h_{i}=\left(h_{i, 1}, \ldots, h_{i, J_{i}}\right)$ is transmitted in slot $\sigma_{j}$ of each epoch of $p$ slots. The reason for restarting each route synchronously at the beginning of each epoch will be explained shortly.

We also require scheduled routes of a system to be compatible in the sense that no two hops, either from the same or different routes, can be scheduled to require transmission from the same node in the same slot. This requirement stems from our assumption that a node can transmit at most once within a slot. The previously stated assumption that all routes are transmitted again in every epoch of length $p$ (instead of, say, each route cycling asynchronously) is designed to permit compatibility to be checked straightforwardly.

In summary a system $\mathcal{S}_{n}=\left\{H_{1}, \ldots, H_{n}\right\}$ for a set of source-destination pairs $\mathcal{P}_{n}$ consists of a compatible set of $n$ scheduled routes, one for each source-destination pair in $\mathcal{P}_{n}$, and with the latest time slot assigned to any hop being defined as the period $p$ of the system. For future use, for $j \in\{1, \ldots, p\}$, let us define the hop set $\mathcal{H}_{j}$ to be the set of hops $(t, r)$ that the system specifies as transmitting in the $j$ th slot. That is, $\mathcal{H}_{j}$ contains a hop $h=(t, r)$ if $h$ is a hop in some scheduled route that is scheduled for the $j$ th time slot. Let us also define the transmission set $T_{j}$ to be the set of nodes that the system specifies as scheduled for the $j$ th slot.

We now describe concretely how a system $\mathcal{S}_{n}=$ $\left\{H_{1}, \ldots, H_{n}\right\}$ with period $p$ for source-destination set $\mathcal{P}_{n}=$ $\left\{\left(s_{1}, d_{1}\right), \ldots,\left(s_{n}, d_{n}\right)\right\}$ transmits data from the sources to the destinations. For each $i \in\{1, \ldots, n\}$, the first packet from $s_{i}$ is transmitted via hop $h_{i, 1}$ in slot $\sigma_{i, 1}$ of the first epoch. Then, if $\sigma_{i, 2}>\sigma_{i, 1}$, this packet is relayed via the second hop $h_{i, 2}$, also in the first epoch. If not, it is transmitted in the second epoch. Subsequently, for each $j>2$, the packet is relayed via hop $h_{i, j}=\left(t_{i, j}, r_{i, j}\right)$ in slot $\sigma_{i, j}$ of the first epoch in which the packet is received at $t_{i, j}$ prior to $\sigma_{i, j}$. Moreover, transmission of subsequent packets from $s_{i}$ to $d_{i}$ are pipelined so that in steady-state one packet from $s_{i}$ is sent over each hop $h_{i, j}$ in each epoch.

\section{B. Success Criteria}

We assume the existence of a transmission success criterion that determines whether or not a given transmission will be successful. Specifically, when a transmitter at $t_{1}$ transmits to location $r$, and the transmission is received at $r$ with power $P_{r, 1}$ in the presence of background noise $N_{o}$ as well as interfering powers $P_{r, 2}, \ldots, P_{r, M}$ from other transmitters, the criteria determines whether this set of received powers permits the transmission from $t_{1}$ to be successfully received at $r$. Such a criterion can be characterized by a success indicator function $\phi\left(P_{r, 1}, \ldots, P_{r, M}, N_{o}\right)$ that takes value one to indicate that the received powers $P_{r, 1}, \ldots, P_{r, M}, N_{o}$ are suitable for a successful transmission, and takes value zero to indicate the opposite. Our specific choice of $\phi$ will be given in the next section.

When a propagation model is available, i.e. a function $\eta(d):[0, \infty) \rightarrow[0, \infty)$ that determines the fraction of transmit power that is received at distance $d$ from the transmitter, then given a set of $M$ locations $T=\left\{t_{1}, \ldots, t_{M}\right\}$ at which there 
are transmitters transmitting with power $P$, the success of a transmission from $t_{1}$ to $r$ becomes a function of $r, t_{1}, \ldots, t_{M}$, as well as $P$ and $N_{o}$. That is, one obtains a success indicator function of the form

$$
\begin{aligned}
& \phi\left(r, T, P, N_{o}\right) \\
& \quad=\phi\left(P \eta\left(\left|t_{1}-r\right|\right), P \eta\left(\left|t_{2}-r\right|\right), \ldots, P \eta\left(\left|t_{M}-r\right|\right), N_{o}\right) .
\end{aligned}
$$

With a transmission success criterion in hand, one may now define a hop set $\mathcal{H}$ to be successful if every hop $(t, r) \in$ $\mathcal{H}$, transmission from $t$ to $r$ is successful in the presence of transmissions from all other transmitters in the transmission set $T$ corresponding to $\mathcal{H}$. Next, one may define a system to be successful if all of its hop sets are successful. Note that in the situation described above in which a propagation model $\eta$ is available, the success of a hop set or a system will depend on $P, N_{o}$ and the propagation model $\eta(d)$, as well as the locations of the transmitters and receivers of the hops in the hop set.

\section{Throughput}

If a system $\mathcal{S}_{n}$ with period $p$ for source-destination set $\mathcal{P}_{n}$ is successful, i.e. if all hop transmissions are received successfully, then in steady-state the system delivers one packet, consisting of $W$ bits, from each source $s_{i}$ to its destination $d_{i}$ in each epoch of $p$ slots. Accordingly, we define the throughput for a successful system to be $\lambda=\frac{W}{p}$. Clearly, to attain large throughput, one needs to design a set of compatible scheduled routes with $p$ as small as possible.

Note that the order in which hops in a route are scheduled has no effect on throughput, though it will effect the delay until the first packets from each source appear at their destinations.

\section{Propagation Models}

Central to throughput scaling studies is the choice of a propagation model, which is a function $\eta:[0, \infty) \rightarrow[0, \infty)$. As mentioned in the introduction, in most of the prior work, e.g. [1], the following signal propagation model (referred to as Propagation Model A) is adopted:

$$
\eta(d)=\frac{1}{d^{\alpha}}
$$

where $\alpha>0$ is a constant whose value depends on the conditions of the channel. Notice that under Model A, when nodes become very close as $n$ becomes large, as happens for example when $\gamma=0$ and the network region $A_{n}$ remains fixed, the received power will be larger than the transmitted, which is not reasonable. In other words, Model A makes sense only as a far field assumption.

This was noted by Arpacioglu and Haas in [8] and by Dousse and Thiran in [9]. In particular, [8] considered the following alternative model (referred to as Propagation Model B):

$$
\eta(d)=\frac{1}{(1+d)^{\alpha}} .
$$

With this model, no matter how close two nodes become, the received power is upper bounded by the transmit power. Similarly, [9] considered a broad class of decreasing propagation models that are upper bounded as $d$ approaches 0 .

\section{SINR AND DistanCE-BASED SUCCESS CRITERIA}

Also central to throughput scaling studies is the question of what constitutes a successful transmission. In particular, we need a criterion to determine when a transmission from $t$ to $r$ will be successful in the presence of other transmitters and background noise. Accordingly, we will adopt the SINR (signal to interference and noise ratio) criterion [1], which is commonly used for this purpose. To introduce it, consider the situation that a set $T=\left\{t_{1}, \ldots, t_{M}\right\}$ of nodes transmit simultaneously in a given slot, that the transmission from $t_{1}$ is intended to be received at $r$, that the received powers at $r$ from the transmitters in $T$ are $P_{r, 1}, P_{r, 2}, \ldots, P_{r, M}$, respectively, and that background noise with power $N_{o}$ is also received. Then the signal to interference noise ratio (SINR) at $r$ is

Definition 1: - SINR

$$
\operatorname{SINR}\left(P_{r, 1}, P_{r, 2}, \ldots, P_{r, M}, N_{o}\right)=\frac{P_{r, 1}}{N_{o}+\sum_{i=2}^{M} P_{r, i}}
$$

Note that with this definition, all other transmissions are considered noise.

\section{Definition 2: - SINR Success Criterion}

Given $\beta>0$, a transmission from $t$ to $r$ is $\operatorname{SINR}_{\beta}$-successful if (and only if)

$$
\operatorname{SINR}\left(P_{r, 1}, P_{r, 2}, \ldots, P_{r, M}, N_{o}\right) \geq \beta,
$$

This criterion is called the physical model in [1].

If a propagation model $\eta(d)$ is available, then SINR becomes a function of the locations of $r$ and the transmitters in $T$, and the powers $P$ and $N_{o}$. Specifically, with a small abuse of notation, let

$$
\begin{aligned}
& \operatorname{SINR}\left(r, T, P, N_{o}, \eta\right) \\
& \quad=\operatorname{SINR}\left(P \eta\left(\left|t_{1}-r\right|\right), P \eta\left(\left|t_{2}-r\right|\right), P \eta\left(\left|t_{M}-r\right|\right), N_{o}\right) .
\end{aligned}
$$

To put this success criterion in the context of the success indicator function introduced earlier, the indicator function is now given by

$$
\phi\left(r, T, P, N_{o}\right)=I\left(\operatorname{SINR}\left(t, r, T, P, N_{o}, \eta\right) \geq \beta\right),
$$

where $I(A)=1$ if $A$ is true and 0 otherwise.

\section{A. Distance-Based Partial Success Criteria}

To design a successful system $\mathcal{S}_{n}$, one must design a set of compatible scheduled routes such that all induced hop sets $\mathcal{H}_{1}, \ldots, \mathcal{H}_{p}$ are successful. While it is straightforward to check if any candidate hop set is successful, it is not at all clear how one goes about designing a hop set to be successful. To facilitate such design, Gupta and Kumar [1] introduced a concept that we refer to as a distance-based success criterion. This is a criterion that can be tested knowing only the distances between the transmitters in a hop set and the various intended receivers. Specifically, Gupta and Kumar first introduced such a criterion called the protocol model, which is specified by two parameters $\rho$ and $\Delta$ and which declares that a transmission from $t_{1}$ to $r$ is successful in the presence of other transmitters in $T$, if $\left\|t_{1}-r\right\| \leq \rho$ and $\left\|t^{\prime}-r\right\| \geq \rho(1+\Delta)$ for all $t^{\prime} \in T$ except $t$. However, in deriving constructive results, [1] used a 
distance criterion of the following form, which we find more useful.

\section{Definition 3: - Distance-Based Success Criterion} $\operatorname{DC}\left(C_{1}, C_{2}\right)$

Given that all nodes in the set $T$ are transmitting simultaneously and given $C_{1}, C_{2}>0$, the transmission from $t_{1} \in T$ to receiver $r$ satisfies $\mathrm{DC}\left(C_{1}, C_{2}\right)$ (and we say $t_{1}, r, T$ is $\operatorname{DC}\left(C_{1}, C_{2}\right)$ successful) if (and only if)

$$
\left\|t_{1}-r\right\| \leq C_{1}
$$

and

$$
\left\|t-t^{\prime}\right\| \geq C_{1}\left(2+C_{2}\right) \text { for all } t, t^{\prime} \in T .
$$

Notice that there is no dependence on power, only on internode distances. Notice also that instead of requiring $\left\|t^{\prime}-r\right\|$ to be large for $t^{\prime} \neq t_{1}$ (as in the protocol model), this criterion requires $\left\|t^{\prime}-t\right\|$ to be large. However, the triangle inequality implies that if $t_{1}, r, T$ satisfies $\operatorname{DC}\left(C_{1}, C_{2}\right)$, then $\left\|r-t^{\prime}\right\|>C_{1}\left(1+C_{2}\right)$ for all $t^{\prime} \neq t_{1}$. Notice also that $\operatorname{DC}\left(C_{1}, C_{2}\right)$ constrains the distance between every pair of nodes in $T$. This has the effect of limiting the density of transmitters, and consequently their total interfering power at $r$.

To use this distance criterion, one must choose $C_{1}$ and $C_{2}$ so that the SINR criterion is satisfied whenever $\operatorname{DC}\left(C_{1}, C_{2}\right)$ is satisfied. No matter how $C_{1}$ and $C_{2}$ are chosen, satisfaction of the SINR criterion depends on the power $P$, the noise power $N_{o}$ and a propagation model $\eta(d)$. Given the latter two items, $N_{o}$ and $\eta(d)$, a pair $C_{1}, C_{2}$ are said to ensure the $\operatorname{SINR}_{\beta}$ criterion, if there exists $P>0$ such that any $t_{1}, r, T$ that satisfies $\operatorname{DC}\left(C_{1}, C_{2}\right)$ also satisfies the $\operatorname{SINR}_{\beta}$ criterion at power $P$, i.e $\operatorname{SINR}\left(t, r, T, P, N_{o}, \eta\right) \geq \beta$. In order to find such $C_{1}, C_{2}$, we prove the following lemma, using techniques similar to those used in [1].

Lemma 1: If $t, r, T$ satisfies $\operatorname{DC}\left(C_{1}, C_{2}\right)$ and $\eta$ is given by Propagation Model B for some choice of $\alpha$, then for any $P>$ 0 ,

$$
\begin{aligned}
& \operatorname{SINR}\left(t, r, T, P, N_{o}, \eta\right) \\
& \quad \geq \frac{1}{\left(1+C_{1}\right)^{\alpha}\left(\frac{N_{o}}{P}+\sum_{k=1}^{K_{n}} \frac{6 k+3}{\left(1+k C_{1}\left(1+C_{2} / 2\right)\right)^{\alpha}}\right)},
\end{aligned}
$$

where $K_{n}=\left\lceil\frac{2 n^{\gamma}}{C_{1}\left(1+C_{2} / 2\right)}\right\rceil$.

Notice that for any $C_{1}$ and $C_{2}$, one can choose $P$ so large that the term $N_{o} / P$ in the denominator of (1) is negligible. We therefore obtain the following.

Corollary 2: $C_{1}, C_{2}$ ensure the SINR criterion if

$$
\left(1+C_{1}\right)^{\alpha} \sum_{k=1}^{K_{n}} \frac{6 k+3}{\left(1+k C_{1}\left(1+C_{2} / 2\right)\right)^{\alpha}}<\frac{1}{\beta},
$$

where $K_{n}=\left\lceil\frac{2 n^{\gamma}}{C_{1}\left(1+C_{2} / 2\right)}\right\rceil$.

The following lemma provides a useful example of $C_{1}, C_{2}$ that ensure the SINR criterion. Among other things, it shows that there is no need to vary $C_{1}, C_{2}$ with $n$.

Lemma 3: For any $\alpha>2, \beta>0$ and $C_{1}>0$, there exists $C_{2}>0$ such that $\left(C_{1}, C_{2}\right)$ ensures the SINR criterion for all $n$.
The next lemma shows that the condition of Corollary 2 cannot be satisfied when both $C_{1}$ and $C_{2}$ are small.

Lemma 4: When $\alpha \geq 2, n \geq(1 / 2+\alpha / 4)^{1 / \gamma}$, and $C_{1}(1+$ $\left.C_{2}\right) \leq 1$,

$$
\begin{aligned}
\left(1+C_{1}\right)^{\alpha} & \sum_{k=1}^{K_{n}} \frac{6 k+3}{\left(1+k C_{1}\left(1+C_{2} / 2\right)\right)^{\alpha}} \\
& \geq \frac{3(2+\alpha)}{(2+\alpha / 2)^{\alpha}} \frac{1}{C_{1}\left(1+C_{2} / 2\right)} .
\end{aligned}
$$

We now provide a converse result, which indicates that if $C_{1}$ and $C_{2}$ become sufficiently small, then $\operatorname{DC}\left(C_{1}, C_{2}\right)$ does not ensure the SINR criterion.

Lemma 5: Given $N_{o}$ and Propagation Model B with parameter $\alpha>0$, and given $C_{1}, C_{2}$ and $n$,

$$
\begin{array}{ccl}
\inf _{T, r, t \text { satisfying } \operatorname{DC}\left(C_{1}, C_{2}\right)} & \sup _{P} & \operatorname{SINR}\left(t, r, T, P, N_{o}, \eta\right) \\
|T| \leq n & \leq \frac{\left(1+n^{1 / 2} C_{1}\left(2+C_{2}\right)\right)^{\alpha}}{4 n^{1 / 2}-12} .
\end{array}
$$

This lemma gives an indication of how SINR can decrease as $C_{1}, C_{2}$ decrease.

\section{THE PRINCIPAL RESUlT}

The following, which is the principal result of this paper, shows that throughput scaling on the order of $\frac{1}{n^{1-\gamma}}$ is asymptotically attainable.

Theorem 6: Consider the many-to-many communication task for a set of $n$ source-destination pairs $\mathcal{P}_{n}$ randomly distributed over a disk of radius $n^{\gamma}, 0<\gamma<1 / 2$, with a propagation model of the form $\eta(d)=1 /(1+d)^{\alpha}$ with $\alpha>2$, an SINR success criterion with parameter $\beta$, and background noise with power $N_{o}$. Then there exists $c>0$ such that for any $n$ and source-destination set $\mathcal{P}_{n}$, there exists a power $P_{n}$ and a many-to-many system $\mathcal{S}_{n}$ with throughput denoted $\lambda(n)$, such that for any packet transmission rate of $W>0$ bits per slot, as $n \rightarrow \infty$

$$
\operatorname{Pr}\left(\mathcal{S}_{n} \text { is } \operatorname{SINR}_{\beta} \text {-successful }\right) \rightarrow 1
$$

and

$$
\operatorname{Pr}\left(\lambda(n) \geq \frac{W}{c n^{1-\gamma}}\right) \rightarrow 1 .
$$

To prove this theorem, we first prove a result like the above, but with the SINR success criterion replaced by a distancebased partial success criterion. The previous theorem will then be proven by appropriate choice of the parameters of the distance-based criterion.

Theorem 7: Consider the many-to-many communication task for a set of $n$ source-destination pairs $\mathcal{P}_{n}$ randomly distributed over a disk of radius $n^{\gamma}, 0<\gamma<1 / 2$, with a distance-based partial success criterion, and packet transmission rate of $W$ packets per slot. For each $n$, let $C_{1}(n)$ be chosen so that

$$
C_{1}(n)<\frac{1}{2} n^{\gamma}
$$

and

$$
n \frac{\frac{C_{1}(n)}{n^{\gamma}}}{\left|\ln \frac{C_{1}(n)}{n^{\gamma}}\right|} \rightarrow \infty, \text { as } n \rightarrow \infty .
$$


Then for any $n$, any $C_{2}(n)>0$, and any source-destination set $\mathcal{P}_{n}$, there exists a constant $c$ and a many-to-many system $\mathcal{S}_{n}$, with throughput denoted $\lambda(n)$, such that as $n \rightarrow \infty$

$$
\operatorname{Pr}\left(\mathcal{S}_{n} \text { is } \mathrm{DC}\left(C_{1}(n), C_{2}(n)\right) \text {-successful }\right) \rightarrow 1
$$

and

$$
\operatorname{Pr}\left(\lambda(n) \geq \frac{W}{c n^{1-\gamma} C_{1}(n)\left(2+C_{2}(n)\right)^{2}}\right) \rightarrow 1 .
$$

Notice that (4) and (5) are rather mild conditions. The first places a natural upper bound on $C_{1}(n)$. For example, when $\gamma=0$, (4) requires that $C_{1}(n) \leq \frac{1}{2}$. The second prevents $C_{1}(n)$ from decreasing too rapidly with $n$. For example, it is satisfied if $\frac{C_{1}(n)}{n^{\gamma}}=\frac{n^{\epsilon}}{n^{1 / 2}}$ for $\epsilon>0$, but not if $\epsilon=0$.

Sketch of the Proof: We follow an approach similar in many respects to that of [1]. The theorem is proven in the following steps.

\section{Step 1: Route selection}

Given $n$ and $C_{1}(n)$, let $z=C_{1}(n) / 2$, it can be shown that the network region region $A_{n}$ can be partitioned into convex cells, each having diameter at most $z$ and area at least $\mu z^{2}$, where $\mu>0$ is some constant that does not depend on $n, \gamma$, or the $z$ 's, and that the number of cells in the partition, denoted $M_{n}$, is at most $\frac{\pi n^{2 \gamma}}{\mu z^{2}}$.

For each $(s, d)$ in the source-destination set $\mathcal{P}_{n}$, draw a straightline line from $s$ to $d$. Form a route for this pair by following the line from $s$ to $d$ and selecting one node for the route from each cell intersected by the line, whenever there is such a node. Convexity of the cells insures that the line does not pass through the same cell twice. The fact that cells have diameter no larger than $z$ implies that if there is at least one node in each cell intersected by the line, then the length of each hop is at most $2 z \leq C_{1}(n)$. Ordinarily, there will be more than one node in a given cell, a fact that can be used to reduce the likelihood that a node is assigned to too many routes. Indeed, if $X_{i}$ source-destination lines intersect the $i$ th cell and this cell contains $Y_{i}$ nodes, we apportion the $X_{i}$ routes as equally as possible among the $Y_{i}$ nodes. Thus, each node is assigned no more than $\left\lceil\frac{X_{i}}{Y_{i}}\right\rceil$ routes. If there are no nodes in $i$ th cell, then we take $\left\lceil\frac{X_{i}}{Y_{i}}\right\rceil$ to be infinity, even if $X_{i}=0$. Let $L(n)$ denote the maximum of $\left\lceil\frac{X_{i}}{Y_{i}}\right\rceil$ over all cells.

Step 2: Potential transmitter sets

We begin by forming a graph with the nodes as the vertices and an edge between any pair of nodes separated by $C_{1}(n)(2+$ $\left.C_{2}(n)\right)$ or less. The degree of node $s_{i}$ is the number of edges connected to it. Let $S(n)$ equal the maximum degree of any node. We now use the graph coloring theorem [10], [11] to assign one of $S(n)$ distinct colors to each node in such a way that no two nodes connected by an edge receive the same color. We then partition the $n$ nodes into the $S(n)$ identically colored groups. Since every pair of nodes in one group is separated by $C_{1}(n)\left(2+C_{2}(n)\right)$, transmissions to receivers located within $C_{1}(n)$ of each will be $\mathrm{DC}\left(C_{1}(n), C_{2}(n)\right)$-successful.

Step 3: System

Step 3 combines the routes of Step 1 and the potential transmitter sets of Step 2 to form compatible scheduled routes, i.e. a system, with period $p_{n}$ no larger than $L(n) S(n)$ and throughput $\lambda(n)=\frac{W}{L(n) S(n)}$. This system will be $\mathrm{DC}\left(C_{1}(n), C_{2}(n)\right)$ successful if all hops have length $C_{1}(n)$ or less, as was the goal of Step 1.

Step 4: Establish two results

In this step we establish the following two results:

(1) $L(n)=O\left(\frac{n^{\gamma}}{C_{1}(n)}\right)$ with high probability, and

(2) $S(n)=O\left(n^{1-2 \gamma} C_{1}^{2}(n)\left(2+C_{2}(n)\right)^{2}\right)$ with high probability.

These two results are proven using the next two lemmas.

Lemma 8: Under the conditions of Theorem 7,

$$
\operatorname{Pr}\left(L(n) \leq c_{1} \frac{n^{\gamma}}{C_{1}(n)}+1\right) \rightarrow 1, \quad \text { as } n \rightarrow \infty
$$

where $c_{1}=2^{14} \pi$.

Lemma 9: Under the conditions of Theorem 7,

$\operatorname{Pr}\left(S(n) \leq \frac{18}{\pi} n\left(\frac{C_{1}(n)\left(2+C_{2}(n)\right)}{n^{\gamma}}\right)^{2}\right) \rightarrow 1$ as $n \rightarrow \infty$.

Step 5: Completion of proof

The system has been designed so that it will be successful provided only that all hops have length $C_{1}(n)$ or less, which, as explained earlier, happens if $L(n) \leq c_{1} \frac{n^{\gamma}}{z}$. Therefore, from Step 4,

$\operatorname{Pr}\left(\mathcal{S}_{n}\right.$ is $\operatorname{DC}\left(C_{1}(n), C_{2}(n)\right)$-successful $) \geq \operatorname{Pr}\left(L(n) \leq c_{1} \frac{n^{\gamma}}{z}\right)$

Where the right hand side goes to 1 as $n \rightarrow \infty$. Since $\lambda(n)=$ $\frac{W}{L(n) S(n)}$ from Step 4, we have that the following quantity goes to 1 as $n \rightarrow \infty$ :

$$
\operatorname{Pr}\left(\lambda(n) \geq \frac{W}{c n^{1-\gamma} C_{1}(n)\left(2+C_{2}(n)\right)^{2}}\right) .
$$

This completes the proof of Theorem 7.

As mentioned earlier, with the above result, Theorem 6 is proven by appropriate choice of the parameters of the distancebased criterion.

\section{REFERENCES}

[1] P. Gupta and P. R. Kumar, "The capacity of wireless networks," IEEE Trans. Inform. Theory, vol. 46, pp. 388-404, Mar. 2000.

[2] M. Grossglauser and D. Tse, "Mobility increases the capacity of ad-hoc wireless networks," INFOCOM, Anchorage, Alaska, Apr. 2001.

[3] E. J. Duarte-Melo and M. Liu, "Data-gathering wireless sensor networks: Organization and capacity," Special Issue Computer Networks (Elsevier) on Wireless Sensor Networks, vol. 43, 2003.

[4] D. Marco, E. J. Duarte-Melo, M. Liu, and D. Neuhoff, "On the manyto-one transport capacity of a dense wireless sensor network and the compressibility of its data," IPSN, Palo Alto, CA, Apr. 2003.

[5] H. El Gamal, "On the scaling laws of dense wireless sensor networks," IEEE Trans. Inform. Theory, 2004, Under submission.

[6] L. Xie and P. R. Kumar, "A network information theory for wireless communication: Scaling laws and optimal operation," IEEE Trans. Inform. Theory, vol. 50, May 2004.

[7] C. Peraki and S. D. Servetto, "On the maximum stable throughput problem in random networks with directional antennas," Proceedings 4th ACM MobiHoc, Annapolis, MD, June 2003.

[8] O. Arpacioglu and Z. Haas, "On the scalability and capacity of wireless networks with omnidirectional antennas," IPSN, Berkeley, Apr. 2004.

[9] O. Dousse and P. Thiran, "Connectivity vs. capacity in dense ad hoc networks," INFOCOM, Hong Kong, Mar. 2004.

[10] G. Chartrand, Introductory Graph Theory, Dover Publications, 1985.

[11] J. A. Bondy and U. S. R. Murty, Graph Theory with Applications, American Elsevier Publishing CO., INC, 1976. 